\title{
Universidad y Democracia
}

Coordinadora:

Mg. Ana Farber

Integrantes del equipo:

Lic. Marisa Boniscontro

Lic. Gladys Elisseche

Es sabido que la desigualdad social presente en las sociedades latinoamericanas se manifiesta en la educación a través de un proceso de selección y exclusión que va creciendo y perfeccionando sus mecanismos, haciéndolos cada vez más sutiles e imperceptibles. Este proceso se inicia desde los primeros años de la escolaridad y recorre toda la educación formal hasta los niveles educativos más altos.

Desde nuestra perspectiva de actores universitarios que no aceptamos como natural esta situación social, el desafío en el que estamos inmersos es justamente poder afrontar la situación y cooperar para su transformación, lo que implica desde nuestra práctica en la universidad desarrollar una tarea académica que nos permita al mismo tiempo articular con la comunidad científica nacional e internacional y atender a las circunstancias propias; generar conocimiento científico y responder a las prioridades sociales; propiciar el fomento de capacidades genéricas para el desempeño individual y social y a la vez estimular el desarrollo de conocimientos específicos para un campo profesional; responder a demandas actuales de trabajo y al mismo tiempo adelantarse e imaginar el mundo futuro que probablemente se sustentará en otros requisitos de formación. En definitiva, asumir, un papel proactivo en la comunidad.

Según diversos estudios realizados para América Latina el quintil más alto de la población es el que asiste a la Educación Superior ${ }^{1}$. Este privilegio es el resultado de las desigualdades económicas, sociales y culturales, que se expresan, como señalábamos, en un trayecto educativo que sin imponer -en la mayoría de los casos- filtros formales va generando exclusión a través de distintas modalidades: estilos organizacionales, códigos para los intercambios educativos selectivos, exigencias de financiamiento familiar muy altas, etc. De este modo, el grupo que ha sorteado exitosamente los filtros, al que Bourdieu ${ }^{2}$

\footnotetext{
${ }^{1}$ Rama, Claudio (2006): La Tercera Reforma de la Educación Superior en América Latina” Fondo de Cultura Económica de Argentina. Buenos Aires.

${ }^{2}$ Bourdieu, Pierre; Passeron Jean-Claude (2003) Los Herederos: los estudiantes y la cultura. Siglo XXI Editores. Buenos Aires.
} 
denomina "Ios elegidos", termina recibiendo una educación "casi exclusiva" que se recubre de una aureola meritocrática. La comprensión de este proceso, profundamente estudiado en el campo de la educación; da cuentas de la presencia de un problema muy grave que pone en jaque el discurso de democratización en la educación superior.

Uno de los motores de la exclusión sin duda es la falta de financiamiento. Es claro, que un sistema educativo que se ha extendido durante los últimos setenta años sin acompañar la creciente cobertura en el nivel medio, con un adecuado incremento de los fondos dedicados a educación ha impactado en la calidad de los aprendizajes. Lo que hace que el acceso al nivel de educación superior, aún en los casos en que es gratuito, imponga restricciones muy fuertes para los sujetos que desean acceder al nivel. La masificación alcanzada en amplias zonas no ha ido acompañada con verdadera políticas de formación y capacitación docente, de ampliación de los recursos pedagógicos (libros, edificios, computadoras, tecnologías, insumos varios) lo que profundizó la segmentación educativa. Así para determinados segmentos educativos que corresponden a los grupos sociales con menor acceso a los bienes económicos y culturales la oferta educativa fue también pobre. El Estado en algunos casos se desentendió y en otros no impactó por llegar con políticas desacertadas frente a las necesidades existentes. Por eso en los segmentos educativos más desfavorecidos, aún en los casos en que se garantizó la retención y finalización de los estudios esto no aseguró a los jóvenes la posibilidad de continuar sus estudios en la universidad. Esa brecha en los aprendizajes que se fue creando producto de la falta de financiamiento en recursos didácticos, tecnológicos, y capacitación docente crea en algunos jóvenes una situación de imposibilidad de acceder a estudios superiores a pesar de haber finalizado la educación media y de poseer los certificados que debieran permitirles el acceso.

Concomitantemente con esta descripción, producto de nuestra experiencia de trabajo con escuelas medias de la zona de influencia de la UNLa, surge en algunos estudios realizados que son los jóvenes procedentes de las escuelas medias privadas los que acceden y ocupan las plazas en las universidades públicas ${ }^{3}$.

Pensamos que esta situación descripta no incluye plenamente la realidad que se manifiesta en las universidades nacionales del conurbano, o que al menos se revirtió con la creación de algunas de ellas.

El hecho de que aparecieran ofertas de educación universitaria gratuita en zonas alejadas de los centros universitarios tradicionales - distancia cultural más que geográfica- traccionó hacia ellas, en un principio, población de diversas edades radicadas en la zona de influencia. Estos, jóvenes que

\footnotetext{
${ }^{3}$ Claudio Rama: ob. cit.
} 
privilegiaban el carácter local de las universidades frente al prestigio que tenían las universidades tradicionales y población de mediana edad que nunca habían iniciado (o no habían podido terminar) sus estudios universitarios, encontraron en el conurbano una oferta educativa motivadora y accesible.

Esta situación, unida a la definición de una política institucional de las UNLa de cooperar con el medio para facilitar el crecimiento tanto de la universidad como de la comunidad local posibilitó la construcción de un modelo alternativo de universidad. En el proceso de construcción de la institución, en el desarrollo de prácticas acordes a los propósitos formulados, en el entramado de tareas y trabajo académico de la universidad, se fue plasmando el nuevo modelo.

La construcción de una nueva universidad en Lanús puso de relieve una compleja combinación de actores e intenciones, implicó no sólo a los alumnos y docentes que transitan por ella, sino también a los ciudadanos y grupos de la comunidad, que comenzaron a interactuar con la universidad generando construcciones simbólicas y nuevos entendimientos de las situaciones e instituyendo nuevas prácticas de vinculación que fueron modificando las tradiciones académicas.

Los modos en que se definieron la organización académica, el proceso de enseñanza, la incorporación de los profesores e investigadores, la articulación con colegas de otras universidades o centros de estudio, con profesionales de otros niveles del sistema educativo, y con los actores sociales y económicos definieron el escenario, materializaron el perfil institucional y generaron nuevas situaciones que pusieron en tensión las tradicionales fronteras académicas.

Al enfrentar situaciones que desbordaban los límites tradicionales de las actividades académicas de las universidades, ya que las actividades reales alcanzaban áreas y aspectos no necesariamente incluidos en la formalización de un solo campo, la flexibilidad organizacional permitió asumir cuestiones complejas a través de variadas combinaciones de actores, poder, autoridad e intenciones.

La intensidad del crecimiento registrado en los diez últimos años de vida académica, el desarrollo del campus universitario, la ampliación de la planta de profesores, el incremento de alumnos, la creación de nuevas ofertas académicas, el desarrollo y la ampliación de las actividades científicas y culturales y de acciones e intercambios con el medio, permite formular un interrogante que quisiéramos responder con esta investigación.

¿De qué forma la vocación democratizadora que se observa con la creación de la universidad y que se expresa en la distribución de la oferta académica así como en una política de intensa articulación con las instituciones, organizaciones y miembros de la comunidad se manifiesta en la actualidad? 
¿Qué cambios se fueron constatando en estos diez años de existencia? ' $̇$ En qué medida el mayor reconocimiento en círculos académicos nacionales e internacionales, el prestigio institucional logrado, y la ampliación de las actividades y redes permiten seguir privilegiando en los intercambios a los sectores que se encuentran en situaciones de mayor debilidad $y / o$ vulnerabilidad social, económica y cultural?

Se fueron generando cambios demográficos en la matrícula universitaria? ¿Qué cambios? ¿A qué factores obedecen? ¿Han logrado acceder a los estudios universitarios los alumnos que no constituyen la "matrícula típica" de las universidades? ¿Qué estrategias se han adoptado desde la universidad para garantizar la permanencia y el egreso de los mismos? La nueva matrícula profesional de egresados, según cada profesión, ha logrado incluirse en el campo profesional con un reconocimiento similar a los egresados de otros centros universitarios de prestigio o por el contrario se ha registrado una menor valoración social de estos egresados? ${ }^{4}$

Para dar respuesta a estos interrogantes el estudio deberá abordar diferentes dimensiones de análisis, una de ellas es la política de ingreso. A diez años de su creación la universidad ha logrado una instalación a nivel nacional, internacional y local, que le aporta un nivel de demanda de vacantes para la formación que ofrece, que supera la oferta académica de gran parte de sus carreras.

Esto genera la necesidad de establecer programas de admisión, que no responden sólo a la necesidad de ajustar la demanda a las posibilidades realistas de oferta académica ya que también cumplen una función propedéutica. Sin embargo, como la ecuación costos=cobertura es siempre desfavorable, si es que no se desea afectar la calidad, al haber más demanda, se incrementan los sistemas de selección.

Pensado en el proceso de diez años de la UNLa, esta situación nos lleva a las siguientes preguntas:

¿Qué modificaciones se perciben en el perfil socio-demográfico de los alumnos aspirantes y de los alumnos ingresantes?

¿Qué estrategias se han desarrollado desde la institución universitaria exclusivamente, desde redes institucionales creadas o existentes, desde otros actores locales para generar políticas positivas de ingreso que aseguren el acceso de matrícula que históricamente no logra ingresar en las universidades tradicionales?

Atravesar satisfactoriamente el examen de selección o curso de admisión, en las universidades que imponen algún tipo de restricción académica o cupos por vacantes para el ingreso es sin lugar a dudas un hecho altamente significativo y seguramente muy auspicioso para el futuro desempeño del estudiante. Sin embargo. En nuestro país no todos los que superan este tránsito difícil entre la

\footnotetext{
${ }^{4}$ Farber, Ana y otros. Nuevas Identidades Profesionales (en evaluación del comité editorial): Esta pregunta ha sido estudiada para describir una modalidad de estudios específicos en la universidad los Ciclos de Licenciatura.
} 
enseñanza media y la universitaria, logran finalizar sus estudios en la universidad. Por eso, en muchos estudios se señala que la asistencia a la educación universitaria no constituye en la Argentina ninguna garantía de finalización.

La información estadística que construye y está disponible en la página web de la Secretaría de Políticas Universitarias del Ministerio de Educación da cuenta de las diferencias existentes entre los aspirantes al ingreso y los ingresantes. En muchos casos, la diferencia se explica por un curso con examen, en otros la diferencia entre inscripción y admisión es inexistente y los escasos alumnos que se pierden se supone que son los que no completaron el proceso de inscripción. Eliminando la información de algunas universidades por inconsistente (número de ingresantes mayor al número de aspirantes) hemos estructurado el siguiente cuadro que presenta diferencias porcentuales muy significativas.

\begin{tabular}{|l|l|l|l|l|}
\hline Institución & Aspirantes & $\begin{array}{l}\text { Nuevos } \\
\text { Ingresantes }\end{array}$ & $\begin{array}{l}\text { Diferencia } \\
\text { valores } \\
\text { absolutos }\end{array}$ & $\begin{array}{l}\text { Porcentaje de } \\
\text { ingresantes }\end{array}$ \\
\hline $\begin{array}{l}\text { Centro de la } \\
\text { PBA }\end{array}$ & 2.793 & 2.118 & 675 & $76 \%$ \\
\hline Chilecito & 507 & 346 & 161 & $68 \%$ \\
\hline Córdoba & 25517 & 18429 & 7088 & $72 \%$ \\
\hline Cuyo & 11816 & 5358 & 6458 & $45 \%$ \\
\hline Sarmiento & 3016 & 705 & 2311 & $23 \%$ \\
\hline La Matanza & 9583 & 3615 & 5968 & $38 \%$ \\
\hline La Rioja & 5217 & 4065 & 1152 & $78 \%$ \\
\hline Lanús & 3135 & 1992 & 1143 & $64 \%$ \\
\hline Litoral & 11203 & 10144 & 1059 & $91 \%$ \\
\hline $\begin{array}{l}\text { Lomas } \\
\text { Zamora }\end{array}$ & 8972 & 6387 & 2585 & $71 \%$ \\
\hline Luján & 5018 & 2654 & 2364 & $53 \%$ \\
\hline Mar del Plata & 8061 & 3993 & 4068 & $50 \%$ \\
\hline Misiones & 5347 & 3698 & 1649 & $69 \%$ \\
\hline $\begin{array}{l}\text { Patagonia } \\
\text { Bosco }\end{array}$ & 3895 & 3516 & 379 & $90 \%$ \\
\hline Quilmes & 4089 & 3674 & 515 & $87 \%$ \\
\hline Rio Cuarto & 3685 & 3353 & 332 & $90 \%$ \\
\hline Rosario & 16972 & 13849 & 3123 & $82 \%$ \\
\hline Salta & 8588 & 4165 & 4423 & $49 \%$ \\
\hline San Juan & 7628 & 4628 & 3000 & $61 \%$ \\
\hline San Luis & 2844 & 2452 & 392 & $86 \%$ \\
\hline Sur & 5337 & 4857 & 480 & $91 \%$ \\
\hline $\begin{array}{l}\text { Tecnológica } \\
\text { Nac. }\end{array}$ & 23043 & 12627 & 10416 & $55 \%$ \\
\hline Tucumán & 17841 & 14560 & 3281 & $82 \%$ \\
\hline Fuente: Proces & & 6 & \\
\hline
\end{tabular}

Fuente: Procesamiento propio en base al cuadro 3.1-2 Aspirantes y nuevos Inscriptos por institución universitaria de gestión estatal. Anuario de estadísticas universitarias 2006.SPU. Argentina 
En países donde el acceso se torna más excluyente por motivos de orden económico básicamente, el esfuerzo de las familias es mayor, y por lo tanto, sólo algunos llegan a la educación universitaria. El carácter abierto o escasamente selectivo de la oferta universitaria, por el contrario, favorece la presencia de muy bajas tasas de egreso en las universidades de gestión oficial.

Claudio Rama señala justamente que los sistemas de admisión abiertos han mostrado menores logros de titulación. La eficiencia de la titulación de Bolivia es del 27,5, de Guatemala el 24,4 y de Uruguay el 28\%. La información estadística para Argentina, según este autor dificulta el poder manejar datos comparativos ya que la flexibilidad permite el abandono y el reingreso lo que alarga extremadamente la duración de los estudios. Por otro lado países con mecanismos de selección de ingreso como Venezuela, Colombia, Brasil, Chile y Costa Rica muestran valores significativamente más altos 48, 57,3; 660,7; 46,3 ; y $46 \%$ respectivamente.

En las universidades argentinas el desgranamiento es muy difícil de medir ya que la condición de estudiante regular varía en cada casa de estudios y el alargamiento de los años de estudios parecería ser una variable importante a considerar para analizar los logros académicos en este nivel de estudios. En el informe estadístico 2006 publicado en 2008 se construye un cuadro que compara la población de ingresantes con los alumnos reinscriptos en dos o más materias y con el total de alumnos. Este cuadro permite observar que los inscriptos por primera vez representan en los casos más extremos el $35 \%$ de la matrícula total de la universidad. Si bien no sabemos si las universidades en cuestión abrieron nuevas carreras y/o tienen carreras de dos o tres años -lo que daría cierta razonabilidad de los números-, el dato alerta sobre un importante desequilibrio en la medida que la mayoría de las carreras universitarias son carreras de cuatro, cinco y seis años de duración.

Este cuadro estaría ofreciendo -con las salvedades del caso- datos estadísticos que reflejarían un proceso de fuerte desgranamiento, fenómeno ampliamente reconocido por quienes actúan cotidianamente en las instituciones universitarias y que impacta desigualmente según el origen social y económico del alumnado.

Para la UNLa en el 2006 los nuevos inscriptos eran 1992, los inscriptos con dos o más materias 4317 y el total de la matrícula 6309. Para ese año había nuevas carreras que se iniciaban y otras que todavía no habían cumplido con el desarrollo de una cohorte completa. Por lo tanto estos datos es necesario reconstruirlos con fuentes propias y más precisas que nos permitan medir más ajustadamente la retención. Del mismo modo la tasa de egreso resulta sesgada por las idénticas razones metodológicas.

Por ello un aspecto importante a considerar dentro de esta línea de investigación es analizar la información estadística existente, producir nuevos procesamientos y cuando lo amerite, producir nueva información si la disponible resulta insuficiente. Ello nos permitirá advertir el tamaño, la intensidad del abandono, identificar ámbitos, momentos en la trayectoria de 
cada cohorte, estacionalidades, condiciones familiares y sociales de la población afectada o en riesgo de ser afectada por el abandono de su proyecto educativo.

Sabemos que si bien todos los alumnos tienen en común que han ingresado a la universidad y son "alumnos regulares" existen diferencias entre ellos, muchas de las cuales incidieron en la elección de la carrera universitaria.

En su libro Los Herederos, Bourdie ${ }^{5}$ plantea que por el sólo hecho de haber ingresado, no se resolvieron las desventajas del pasado social de los alumnos provenientes de los sectores socioeconómicos más pobres. Estas desigualdades se transforman en déficts educativos por el juego de mecanismos sustitutos como lo son las orientaciones precoces 0 mal informadas, las elecciones forzadas o los estancamientos durante la cursada. Basil Bernstein ${ }^{6}$ analizando otros niveles educativos se refiere a los problemas de enmarcamiento y de códigos que difieren entre las instituciones educadoras y la cultura de los alumnos procedentes de sectores sociales pobres. Las posibilidades de adecuación e interacción con los esquemas de enseñanza y trabajo académico que se propone desde la universidad no son iguales para todos los alumnos, y esto afecta el rendimiento académico determinando el éxito o el fracaso de los estudiantes.

Muchas veces la elección de una carrera responde a una predisposición socialmente condicionada, ya sea porque sostener esos estudios cuesta caro, porque para desempeñar la profesión se requiere un patrimonio que no se tiene, porque no coincide con las representaciones del trabajo femenino, etc. Todos estos aspectos que intervienen en las decisiones iniciales, generan un estilo de integración e interacción académica; son disposiciones y saberes que los alumnos construyeron en su medio y que constituyen entramados personales desde los cuales se definen elecciones y se acomodan y estructuran los nuevos aprendizajes propios de sus estudios en la universidad.

Toda enseñanza supone, siguiendo a Bourdieu, un cuerpo de saberes, no todos escolares, saber hacer y saber decir constituyen patrimonios culturales ampliamente desarrollados en los sectores sociales más favorecidos.

En su obra Los Herederos, señala que asegurar a través de un sistema de becas "los medios económicos a aquellos que tienen los "dones" indispensables es quedarse a mitad de camino en el análisis de los obstáculos e ignorar que las aptitudes medidas con el criterio educativo se deben más que a los dones naturales, a la mayor o menor afinidad entre los hábitos culturales de una clase y las exigencias del sistema de enseñanza o los criterios que definen el éxito en él" (Bourdieu: 2003, pág.38)

\footnotetext{
${ }^{5}$ Bourdie, Pierre. Ob. cit

${ }^{6}$ Bernstein, Basil: (1998) Clases, códigos y control I, II, III, IV. AKAL Universitaria. Madrid España
} 
Esta afirmación no niega el valor de la compensación que puede estimular a un estudiante al sentirse más respaldado económicamente pero a la vez no agota la complejidad de dimensiones del problema de las desventajas académicas y por lo general no incluye otro tipo de compensaciones y puentes que debieran plantearse dentro de la propuesta de trabajo académico.

Estas desventajas se expresan de diversos modos: el estancamiento -envejecimiento y retraso en los estudios, diferencias de actitud y aptitud por obstáculos económicos y culturales, distintas expectativas subjetivas al acceder a la universidad, progresar e identificarse con el futuro académico y/o profesional.

Resulta una distinción importante para el análisis de las desventajas académicas la distinción (Bourdieu: 2003 ) entre la facilidad para asimilar la cultura transmitida y la propensión a adquirirla.

La propensión se vincula con el medio familiar, la incitación al esfuerzo permite compensar la desposesión con la aspiración. Muy laboriosamente el alumno de sectores sociales más bajos puede apropiarse de las ventajas sociales que aunque imperceptibles son siempre acumulativas. En efecto los estudiantes más desfavorecidos pueden encontrar en la cultura académica, en las conductas académicas (lectura, música, espectáculos, cine) medios de compensar sus desventajas.

Las acciones tendientes a asegurar la continuidad de los que ingresaron deben incluir el acercamiento a esos saberes que no están dentro de las propuestas de formación académica, pero que son imprescindibles para participar activamente de la vida social y además lograr el desempeño profesional exitoso. Debe apropiarse durante su trayecto universitario de la cultura social acumulada que le permita saber hacer y saber decir como sujeto social competente desarticulando la exclusión de la que fue objeto $y$, tendiendo puentes desde la experiencia y el esfuerzo realizado para que cada vez sea más accesible y más cercana su comunidad de origen y la comunidad universitaria.

Si esto se logra construir, se favorece las buenas inserciones en el trabajo y la movilidad laboral de los egresados porque en la apropiación de la cultura social acumulada se incorporan competencias y recursos para participar de espacios y redes sociales y profesionales.

"Para los individuos provenientes de los sectores más desfavorecidos, la educación sigue siendo el único camino de acceso a la cultura y esto en todos los niveles de enseñanza. Podría ser entonces la vía regia de la democratización de la cultura si no se dedicara a consagrar - por el simple trámite de ignorarlas- las desigualdades iniciales ante la cultura y si no soliera llegar - por ejemplo reprochándole un trabajo académico que sea demasiado académico" - hasta desvalorizar la cultura que se transmite en beneficio de la 
cultura heredada que no lleva la marca del esfuerzo y, de ese modo, favorecer a quienes aparentan facilidad y gracia.( Bourdieu 2003:pág. 37).

La posibilidad de enfrentar esta situación estaría, entonces en la capacidad de las instituciones universitarias de compensar las desventajas distribuyendo y construyendo "capital social". Entendiendo el concepto de capital social como un agregado de recursos personales, actuales y potenciales, que están vinculados a la posesión o acceso a una red permanente de relaciones más o menos institucionalizadas de mutua aceptación y reconocimiento. Para Bourdieu el capital social no sólo está representado en las relaciones sociales que un individuo puede usar para aspirar al acceso de recursos de aquellos con los cuales está conectado; sino también está contenido en la cantidad y calidad de dichos recursos. James Coleman ${ }^{7}$ incorpora el término capital social a las ciencias sociales, identificándolo con la capacidad de las personas para trabajar en grupos, en base a un conjunto de normas y valores compartidos. Para Coleman los valores del capital social se presentan en el plano individual, fortaleciendo la capacidad personal para relacionarse mediante redes de contactos sociales que se fundamentan en expectativas de reciprocidad y comportamiento confiable que, en conjunto, mejoran la eficiencia individual contribuyen a configurar conductas cooperativas y acciones colectivas de beneficio mutuo.

El capital social que la universidad construye y distribuye, forma parte de los aspectos menos visibles de la formación académica y permite compensar las desigualdades de origen de los alumnos procedentes de grupos con mayores deventajas sociales con propensión a adquirir la cultura.

La propensión a adquirir las competencias sociales y los saberes académicos es desigual entre los estudiantes con menores pronósticos de éxito y se manifiesta en diferentes actitudes frente a las dificultades académicas: persistencia, obstinación o abandono. El compromiso del educador en cualquier nivel de enseñanza que se trate es el de impedir que estas actitudes y definiciones individuales sean invisibles y actuar dando respuestas adecuadas y diversificadas. Asumir esta perspectiva pone en juego el "ethos" de la institución, porque siempre se trata de hacer cosas distintas, tensar modelos instituidos, cuestionar legalidades establecidas.

La propensión a estudiar está en relación directa con la valoración de la cultura como fin y como medio de crecimiento. Esta es una construcción individual que se sustenta en lo lazos que el sujeto crea con otros sujetos (padres, maestros,

${ }^{7}$ COLEMAN, JAMES, "Social Capital in the Creation of Human Capital". American Journal of Sociology, 99, (Supplement):S95.S120,1988 
otros adultos, ídolos populares) y objetos (libros, discos, instrumentos musicales, películas) desde los primeros años de su vida.

Esta propensión puede ser vista como posibilidad, como recurso personal que diferencia al sujeto de su entorno empobrecido y que lo proyecta a cambiar de entorno o, en el mejor de los casos, cambiar el entorno ubicando a la educación como una vía para hacerlo.

Esta visión de futuro difiere de la inmediatez, el exitismo y la búsqueda de la satisfacción inmediata que transmites permanentemente los medios de comunicación y una parte del arte popular.

En la práctica educativa (entendiendo por esta el conjunto de prácticas institucionales) se produce el encuentro que potencialmente puede romper el círculo de reproducción de las condiciones iniciales desfavorables de algunos alumnos, este es el encuentro entre la propensión a estudiar y crecer y la pasión por enseñar en un contexto que asegure los recursos y las condiciones adecuadas para desarrollar con plenitud estas actividades.

Primeras preguntas de investigación:

Al tradicional subtítulo "preguntas de investigación" le adiciono primeras, porque la naturaleza del enfoque metodológico múltiple al que haré referencia me permite suponer que si bien algunas preguntas van a sostener su enunciación hasta el final del proceso de investigación, otras por la naturaleza más cualitativa de su aproximación al objeto van a ir logrando mayor nivel de especificación y distinción en la medida que se incremente el conocimiento sobre el objeto.

- Cómo se ha ido construyendo la población estudiantil de la UNLa y cuáles son sus sistemas de preferencias académicas

- Cuáles son los factores que intervienen en la determinación de la elección de una carrera

- Se puede inferir un sistema de predicciones en los logros académicos partiendo de las características socio-demográficas de la población estudiantil.

- Si tal situación es posible cuáles son las características de los alumnos que no cumplen con la predicción estadística?

- De este grupo, cómo son los alumnos que proveniendo de sectores socioculturales más vulnerables logran llegar al final de su carrera? 
- Cuáles fueron los recursos personales, familiares, institucionales que intervinieron como facilitadores y cuáles los obstáculos al aprendizaje, que encontraron durante su trayectoria estudiantil.

- Qué componente de la formación recibida como parte de la propuesta académica y de la vida universitaria le resulta o le resultó más valiosa para ampliar sus marcos referenciales y redes de intervención.

\section{Metodología:}

Las preguntas formuladas requieren un abordaje múltiple. Hay algunas cuya respuesta nos orienta a estrategias metodológicas cuantitativas, mientras que otras nos plantean abordajes cualitativos.

Por ello, se piensa trabajar con diferentes fuentes, Información estadística y documental,

entrevistas en profundidad y focus grup.

Para las técnicas cualitativas se utilizará una muestra teórica de carácter intencional, no obstante la misma podrá ser expandida si no logra satisfacer el criterio de saturación teórica de Glasser y Strauss ( la recolección finaliza cuando una nueva entrevista o un nuevo documento ratifica lo ya recogido, sin incorporar nada nuevo). Este nos parece un criterio muy potente para la investigación cualitativa, porque persigue la reconstrucción de las representaciones que organizan y dan sentido al comportamiento social respecto de un determinado hecho.

Bibliografía:

ABRAHAM, ADA: (1998) La identidad profesional de los docentes y sus vicisitudes, en Revista del Instituto de Investigaciones en Ciencias de la Educación. Miño y Dávila Ball, Stephen J. (1989). "La micropolítica de la escuela. Hacia una teoría de la organización escolar". Paidós. España, Barcelona.

Berger, Peter y Luckman, Thomas, (1986): La construcción social de la realidad. Editorial Amorrortu. Madrid

Bernstein Basil: (1997) La Estructura del discurso pedagógico; Clases, códigos y control (volúmen IV) Ediciones Morata. Madrid. 
Bernstein Basil:(1990) Poder, educación y conciencia. Sociología de la transmisión cultural, El Roure Editorial, Barcelona.

Birgin, Alejandra(1997) El trabajo de los profesores en Gentile, Pablo Y Frigotto, Gaudencio coords, La ciudadanía negada; políticas de exclusión en la educación y el trabajo, CLACSO, Buenos Aires.

Bourdieu, Pierre: (1997) Capital cultural, escuela y espacio social. Siglo XXI Editores,Madrid.

Bourdieu, Chamboredon, Passeron. (1998) El oficio del Sociólogo. Siglo XXI. México.

Bourdieu, Pierre: (1999) Razones prácticas, sobre la teoría de la acción. Anagrama, Barcelona. España.

Editores. Argentina, Buenos Aires

ABBOTT, A. (1998), The System of Professions. An essay on the Division of Export Labor. University of Chicago Press. Chicago.

ADORNO THEODOR: (1992), DIALÉCTICA NEGATIVA, TAURUS, $5^{\circ}$ EDICIÓN, MADRID

ADORNO, THEODOR (1998): EDUCACIÓN PARA LA EMANCIPACIÓN, MORATA, MADRID

APPLE, MICHAEL: (1996) Política cultural y educación, Ediciones Morata, Madrid.

APPLE MICHAEL, BEANE JAMES (1997) Escuelas democráticas. Ediciones Morata. Madrid.

BALL, STEPHEN J. (1989). "La micropolítica de la escuela. Hacia una teoría de la organización escolar". Paidós. España, Barcelona.

BERGER, PETER Y LUCKMAN, THOMAS, (1986): La construcción social de la realidad. Editorial Amorrortu. Madrid 
BERNSTEIN BASIL: (1997) La Estructura del discurso pedagógico; Clases, códigos y control (volúmen IV) Ediciones Morata. Madrid.

BERNSTEIN BASIL :(1990) Poder, educación y conciencia. Sociología de la transmisión cultural, El Roure Editorial, Barcelona.

B BOURDIEU, PASSERON. (1998): Los Herederos Siglo XXI. México. BOURDIEU, PIERRE : (1999) Razones prácticas, sobre la teoría de la acción. Anagrama, Barcelona. España.

BOURDIEU, PIERRE : (1997) Capital cultural, escuela y espacio social. Siglo XXI Editores,Madrid.

CARR, W Y KEMMIS, S: (1988) Teoría Crítica de la Enseñanza. Marténez Roca. Barcelona.

CHARTIER, ROGER (1996) El Mundo comorepresentación. Historia cultural: entre práctica y representación. Madrid, Barcelona, ( $3^{a}$ edición) CONNELL, ROBERT: (1997) Escuelas y justicia social. Ediciones Morata. Madrid.

Cox Cristián: Clases, reproducción cultural y transmisión escolar: una introducción a las contribuciones teóricas de Bourdieu y Bernstein.

DE CERTEAU, MICHEL. (1999). La cultura en plural. Ediciones Nueva Visión. Buenos Aires, Argentina.

DURKHEIM, E. (1967). De la división del Trabajo Social: Schaipire, Bs.As.

GAUCHET, MARCEL. (2004). La democracia contra sí misma. Ediciones HomoSapiens. Santa Fe, Argentina.

GIMENO SACRISTÁN, JOSÉ: (1998) Poderes inestables en educación. Morata, Madrid.

GIROUX HENRY: (1997) Cruzando límites. Paidós Educador. Buenos Aires. 
GIROUX HENRY (1999). Los profesores como intelectuales. Hacia una pedagogía crítica del aprendizaje. Editorial Paidós/MEC. Madrid, España.

GRAMSCI, ANTONIO: (1985) La alternativa pedagógica. Hogar del Libro. Barcelona.

GRIGNON, CLAUDE Y PASSERON,JEAN-CLAUDE: (1989). Lo culto y lo popular. Miserabilismo y populismo en sociología y en literatura. Ediciones Nueva Visión. Buenos Aires, Argentina.

GRUNDY, S: (1994) Producto o praxis del curriculum. Ediciones Morata. Madrid.

MARX, K. y ENGELS, F.(1985) La Ideología Alemana, Ediciones Pueblos Unidos, Buenos Aires.

PARSONS, T. (1959) "The Professions and the Social Structure".Essays in Sociological Theory. New York .

PARSONS, T. (1969) El Sistema Social, CEFyL, UBA, Buenos Aires. PERRUSI, A (2000): Profesión, vocación y medicina. Revista Política. Trabajo 16

STRAUSS A. y CORBIN J.(1994), Grounded Theory Methodology en Handbook of Qualitative Research, Sage, Londres (traducción en español sin autor)

WEBER, M. (1969)La ética protestante y el espíritu del capitalismo. Editorial Península Barcelona.

WEBER, M. (1993)Economía y sociedad. Madrid: Fondo de Cultura Económica, Madrid.

WEBER, M. (1986) El político y el científico. México: FCE. 